\title{
Inmigrantes en las series de televisión Aída y La que se avecina. Entre la parodia y los prejuicios
}

Q institucionales.us.es/ambitos/

Ana Isabel Abad Villamor

Universidad San Jorge

anaisabelabadvillamor@gmail.com

\section{Cayetano Fernández Romero \\ Universidad San Jorge \\ cfernandez@usj.es}

English Version: Inmigrants in the television series Aída and La que se avecina. Between parody and prejudice

\begin{abstract}
Uno de los fenómenos más impactantes y noticiosos en España en las últimas décadas ha sido el número de personas extranjeras llegadas en pocos años y su impacto en el ámbito sociodemográfico, económico y cultural. Este proyecto pretende analizar el tratamiento de dicho fenómeno en los medios a través del análisis de los personajes inmigrantes de dos series de televisión de gran audiencia en España: Aída y La que se avecina. Los resultados exponen, más allá de la parodia, una visión crítica del trato de los españoles hacia los inmigrantes y de la representación estereotipada de la inmigración
\end{abstract}

Palabras clave: series de televisión, inmigración, humor, estereotipos.

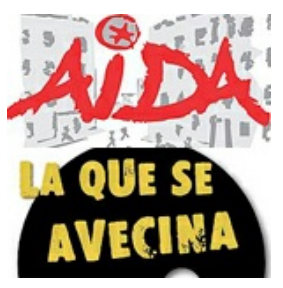

Abstract: One of the phenomena which have caused most impact in Spanish media during the last decades has been the influx of immigrant populations and their contribution on all areas of society, from the strictly demographic to the economy and culture. In this project we aim to analyse the treatment of this phenomenon in the media, above all, through the analysis of the profile of immigrants of two of the highest audience rated TV series in Spain; Aída and La que se avecina. The results go beyond mere parody providing a critical vision of how the Spanish treat immigrants and offering a stereotyped representation of immigration.

Keywords: television series, immigration, humour, stereotypes.

\section{INTRODUCCIÓN Y OBJETIVOS}

Uno de los fenómenos demográficos más llamativos que ha vivido España en las últimas décadas ha sido la llegada de inmigrantes. No por ser un proceso novedoso. Lo realmente destacable es el número de personas extranjeras llegadas en pocos años. Pasando, por ejemplo, de representar el 7\% de la población en España en 2004 a algo más del $12 \%$ desde el año 2009 y mostrando un crecimiento ininterrumpido hasta el año 2011. Sin embargo, la crisis ha incidido en este comportamiento y a partir de dicha fecha se observa una tendencia decreciente del número total de inmigrantes en España, según los datos del Padrón Continuo publicados por el Instituto Nacional de Estadística (INE, 2014).

Por otro lado, ni la incidencia de este proceso ni el porcentaje de migrantes por nacionalidades son homogéneos según los datos del INE [1]. Los ecuatorianos, que representaban la primera comunidad de inmigrantes ya que constituía el $15 \%$ en 2005 , han descendido a un $5,3 \%$ en 2013 . También ha habido un descenso de un $8 \%$ a un $4 \%$ en la inmigración colombiana. En la población inglesa ha ocurrido la situación inversa, de un $5,8 \%$ ha pasado a un 6,9\% en las mismas fechas. En cambio, otras nacionalidades como la marroquí han mantenido un porcentaje de población importante en España a lo largo del periodo con porcentajes superiores al 12,4\%. Por su parte, la población rumana se ha multiplicado por dos en estos años, llegando a constituir más del $12 \%$.

En este contexto, hay que tener en cuenta que las migraciones no son acciones individuales en la que una persona decide trasladarse a otra región dejando atrás sus arraigos culturales y asimilándose rápidamente al nuevo país de destino. Más bien consiste en un complejo y largo proceso que afectará también a las generaciones siguientes, transformando desde el punto de vista social, político y económico tanto a la sociedad emisora como a la receptora (Castles, De Haas y Miller, 2014).

En esta última, uno de los principales cambios ha sido el aumento de la diversidad cultural y socioeconómica, lo que ha generado la necesidad en las sociedades receptoras de llevar a cabo una profunda reflexión sobre cuál es la manera más adecuada de gestionar esta diversidad (Innerarity y Acha, 2010). Los medios de comunicación, que juegan un rol muy importante en la construcción de la opinión pública (Lévi-Strauss, 1965; Ramos, 1995), se convierten así en un objeto clave de estudio debido a la importancia que supone el imaginario colectivo en la garantía de cohesión social. Por este motivo, numerosos expertos han analizado el discurso que los medios de comunicación españoles han aportado sobre la cuestión migratoria, y qué reflexión sobre el fenómeno subyace a dicho mensaje (Lirola, 2013; Van Dijk, 1997; Bañón, 2002; Rodrigo y Medina, 2009).

El presente trabajo se centrará en las investigaciones de aquéllos que analizan el discurso audiovisual, y más en concreto, la ficción televisiva. Esta investigación se planteó como punto de partida de un proyecto más amplio, una tesis doctoral, cuyo principal propósito ha sido averiguar qué mensaje sobre la inmigración está recibiendo la población adolescente, que consume mayoritariamente series de ficción (Medrano, Aierbe y Palacios, 2007; Figueras, 2009). La elección de los adolescentes se debe a que el poder de penetración cultural de los medios tiene más influencia en personas que están viviendo una etapa clave de construcción de su identidad (Coleman, 1985; Perinat, 1997; Erikson, 1968). Y en el caso de la ficción seriada, los adolescentes la consumen como "alimento anímico" que amplía y organiza su vivencia psíquica y afectiva, contribuyendo a crearles nuevas formas de interacción en la sociedad y nuevas maneras de relacionarse con los otros y consigo mismos (García de Castro, 2007; Durkin, 1985; Montero, 2006).

Así, el trabajo aquí presentado tiene como finalidad analizar el discurso que las series de ficción transmiten en España sobre el fenómeno de la inmigración, con el fin último de conocer qué mensaje se está proyectando sobre la población, especialmente los jóvenes, y de qué manera podría influir en su construcción simbólica de la realidad; o como afirman González de Garay y Alfeo (2012: 941): “identificar, describir e 
interpretar esos prejuicios" (en referencia a la inmigración) y "analizar cómo las ficciones televisivas los utilizan, lo que resulta esencial para comprender sin ensimismamientos ciertos procesos sociales y culturales".

Existen numerosos trabajos que ofrecen múltiples perspectivas al respecto. Por un lado, los inmigrantes aparecen en un contexto de irregularidad e ilegalidad, tienden a la trampa y el engaño, siempre son representados de forma negativa y se asocian a problemas (Galán, 2006; Ruiz Collantes et al., 2006; Igartua et al., 2012; Lacalle, 2008).

Por otro lado, en algunas ocasiones estas características estereotipadas van asociadas a un tratamiento sarcástico y humorístico de la realidad, como es el caso de las comedias de situación o sitcom. Se afirma que "la utilización del humor es un mecanismo representacional para ridiculizar una realidad absolutamente grave como es el racismo", pero que al final los tópicos y estereotipos calan en los telespectadores porque se asientan sobre ideas presentes en la sociedad (González de Garay y Alfeo, 2012: 940). Medina, Concepción y Rodrigo (2008) también se plantean si la presencia en los guiones de valores negativos sobre la inmigración perpetúa los prejuicios, o si la ironía puede llevar precisamente a la concienciación social y a romper con los estereotipos sociales. En esta disyuntiva trabajan Biscarrat y Meléndez (2014) a propósito de la serie Aída. En cualquier caso, coinciden con el hecho de que se observa una omnipresencia de estereotipos y que, a pesar de las características humorísticas del género, "de manera casi perversa, la ficción televisiva contribuye a la producción y mantenimiento de estereotipos etno-raciales" (Biscarrat y Meléndez, 2014: 342).

En definitiva, de los diferentes trabajos analizados sobre series de ficción en el contexto español se han extraído las siguientes características de los personajes inmigrantes, sintetizadas en nueve categorías: humor, irregularidad (personas en situación irregular o ilegal), violencia (asociada al crimen y los delitos), baja cualificación (menor preparación desde el punto de vista académico), infrarrepresentación (no son protagonistas), engaño, racismo, falta de evolución del personaje y tópicos.

A tenor de lo expuesto, en este trabajo se han analizado las series más visionadas y preferidas por los adolescentes, que como ya se ha comentado constituyen la franja de población objeto de estudio. En este caso, como primer punto de partida se realizó una encuesta sociomediática a un grupo de 236 jóvenes entre 14 y 16 años de la ciudad de Zaragoza, estudiantes de cuatro centros educativos seleccionados por los diferentes porcentajes de alumnado autóctono e inmigrante [2]. En los resultados destacan La que se avecina, señalada por el 63\% de los adolescentes, y Aída (46\%). Por otro lado, hay que mencionar que ambas series han obtenido excelentes audiencias a lo largo de los años:Aída un «share» de media de un 20.6\%; y La que se avecina un 21\% (datos de la página web Fórmula TV).

A partir de estos datos y la teoría expuesta anteriormente, se plantean los siguientes objetivos en este proyecto:

-Describir el perfil de los personajes inmigrantes que aparecen en las series Aída y La que se avecina; detallar el papel de los personajes autóctonos en relación a estos primeros; y examinar si dicho perfil coincide con los rasgos presentados en las investigaciones anteriormente comentadas.

-Exponer las similitudes y diferencias entre las dos series objeto de estudio con respecto al primer objetivo.

-Analizar qué lectura puede extraerse de esa representación teniendo en cuenta que son comedias de situación (sitcom).

\section{METODOLOGÍA}

Para la consecución de estos objetivos, son necesarias técnicas como el análisis de contenido que nos permitan describir el mensaje (Andréu, 2001; McQuail, 2000), metodología que hemos llevado a cabo en este proyecto desde la perspectiva cualitativa. El análisis de contenido cualitativo, también llamado por algunos autores análisis categorial (Bardin, 1977; Fiske, 1987), hace referencia a las operaciones de descomposición del texto en unidades, y a partir de ahí, a la clasificación de las unidades en categorías.

En este estudio, el análisis de los datos sigue un modelo inductivo de agrupación temática, apoyándose en las categorías previas propuestas por la bibliografía (Ryan y Bernard, 2003; McMillan y Schumacher, 2005). En otras palabras, hemos comprobado si están presentes las nueve categorías que se especifican anteriormente; y, por otro lado, mediante el modelo inductivo, hemos agrupado los datos en categorías emergentes.

En cuanto a las fuentes, se han visionado y transcrito 18 capítulos de las dos series, aproximadamente 1.840 minutos. Por un lado, enLa que se avecina, que ya ha emitido ocho temporadas completas y se está emitiendo actualmente la novena, se han seleccionado nueve capítulos en los que aparece el principal personaje inmigrante de la serie, Rosario Parrales, y de forma puntual su madre, ambos colombianos. Los capítulos han sido elegidos por ser los más vistos de cada temporada según datos de Fórmula TV $(4 \times 05,4 \times 11,4 \times 12,5 \times 10,5 \times 11,5 \times 12,6 \times 01,6 \times 02,6 \times 03)$. Además, se han transcrito otros episodios que aparecen destacados en la página web oficial de la serie, en el apartado dedicado a Parrales.

Con respecto a la serie Aída, que tiene en total 10 temporadas, el corpus se compone también de nueve capítulos. En cada uno de ellos, hay una trama principal que gira en torno a un personaje inmigrante, además de contar con buenos índices de audiencia (Fórmula TV). El que mayor protagonismo tiene es el ecuatoriano Osvaldo, alias Machupichu $(5 \times 02)$. En segundo lugar, se encuentra el argentino Néstor, alias Aconcagua $(10 \times 38)$. El resto de personajes son esporádicos: el novio cubano de la abuela Eugenia, Oswaldo (1×03); la profesora inglesa Mary $(2 \times 10)$; el estadounidense chino-americano Bobby Tang (4×05); el padre del hijo de la joven Macu, el senegalés David (7×10); los estudiantes suecos Stig y Lars (8×14); el soldado inglés Brandon (9×14); y el novio saudí de la joven Lorena, Omar (9×18).

\section{ANÁLISIS Y RESULTADOS}

Como ya se ha comentado, en este trabajo el análisis consiste en agrupar los datos temáticamente. Por ello, nos basaremos en las nueve categorías propuestas por la bibliografía, pero completando la información con otras ocho categorías emergentes resultado del análisis de las dos series: amistad, amor, formación educativa, solidaridad, subordinación, chantaje, machismo y utilitarismo. En definitiva, trabajaremos con 17 categorías como se puede observar en la tabla 1. Para facilitar el establecimiento de relaciones y su posterior análisis, estas 17 categorías han sido agrupadas en cinco macrocategorías (McMillan y Schumacher, 2005). 


\begin{tabular}{|c|c|c|c|c|}
\hline Actos & $\begin{array}{l}\text { Significados } \\
\text { asociados } \\
\text { Lenguaje }\end{array}$ & Participación & Relaciones & Contextos \\
\hline $\begin{array}{l}\text { Racismo* } \\
\text { Violencia } \\
\text { Utilitarismo } \\
\text { Machismo } \\
\text { Engaño } \\
\text { Chantaje } \\
\text { Subordinación } \\
\text { Solidaridad }\end{array}$ & $\begin{array}{l}\text { Racismo* } \\
\text { Tópicos } \\
\text { Humor }\end{array}$ & $\begin{array}{l}\text { Evolución del } \\
\text { personaje } \\
\text { Infrarrepresentaci } \\
\text { ón }\end{array}$ & $\begin{array}{l}\text { Amistad } \\
\text { Amor }\end{array}$ & $\begin{array}{l}\text { Baja } \\
\text { cualificación } \\
\text { Formación } \\
\text { educativa } \\
\text { Irregularidad }\end{array}$ \\
\hline
\end{tabular}

Tabla 1. Marco conceptual de categorías para el análisis de la representación migratoria en Aída y La que se avecina.

La definición de las macrocategorías se inició creando constructos abstractos que permitieran relacionar teóricamente las distintas categorías, para lo cual nos ayudamos de la clasificación de códigos que plantea Lofland (1971) para el análisis textual de datos cualitativos: actos, significados, participación, relaciones y contextos. Los Actos son las acciones de los distintos personajes. Los Significados hacen referencia a las producciones verbales de los personajes, de ahí la puntualización del lenguaje. En estas dos macrocategorías hay una categoría que se repite, el Racismo, que como veremos más adelante es la idea más profusa y con mayor proyección en ambas series. La Participación es la tercera macrocategoría, que en este estudio en concreto implica la adaptación de un personaje a las tramas, así como el protagonismo que se le otorga. Por otro lado, los vínculos emocionales entre personajes son considerados Relaciones (Lofland, 1971). Y, por último, la macrocategoría Contextos se ha inspirado en la categoría previa Irregularidad. Abordaremos cada una de ellas, junto a los resultados, en los siguientes apartados.

\subsection{Actos}

La macrocategoría denominada Actos hace referencia a la forma de actuar de los distintos personajes con los demás. En este bloque hay ocho categorías a tener en cuenta. La más importante y que mayor proyección ha tenido es el Racismo, que se puede vincular directa o indirectamente con el resto de categorías. Menos una categoría, todas tienen una connotación negativa.

\subsubsection{Racismo}

El Racismo es un atributo muy presente en las series analizadas. Los personajes a los que se puede asociar esta característica son Mauricio Colmenero (el actor Mariano Peña) en Aída, dueño del bar Reinols; y Antonio Recio (Jordi Sánchez) en La que se avecina, dueño de una pescadería. Estas dos personas representan el perfil de autóctono de ideología conservadora, racista, machista y homófoba. Y tienen en común, además de estos rasgos, el hecho de haber contratado a inmigrantes para trabajar en sus respectivos negocios.

Como ejemplos paradigmáticos de Racismo en el caso de Mauricio («Aída»), podríamos destacar: el momento en que no les quiere solucionar la inundación del piso que les alquila porque "éstos ya están hechos a la humedad. No ves que cruzaron el Mediterráneo en cayuco" (5×02); todos los instantes en que les pega con un estropajo o un paquete de folios en la cara cuando han cometido un error, o simplemente por divertirse, a la vez que insulta su procedencia y sus características culturales; cuando les obliga a cometer delitos menores para conseguir un objetivo personal y no respeta su horario de trabajo ni su salario; al no permitir (o al menos intentarlo) que Osvaldo tenga una relación personal con su sobrina; entre otros muchos.

Antonio Recio (La que se avecina) no utiliza tanta violencia física como su homólogo en Aída, pero es un experto manipulador que engaña y chantajea a su empleado Parrales constantemente, y en muchos casos no lo hace por dinero, sino por ideología. Un ejemplo de ello son los distintos momentos en que intenta boicotear la relación de Parrales con su novia española y después mujer María del Carmen (María Bazán) por considerar que "está en juego la pureza de nuestra raza" (episodio "La madre de Parrales, una mula").

Estar explotado laboralmente, cometer delitos menores e incluso llegar a disfrazarse de quisquilla (como si fuera una mascota) también son factores que Parrales debe soportar.

Dentro de estas similitudes en el trato a los inmigrantes, hay una distinción importante entre ambas series, y es el hecho de que Osvaldo de Aída, a diferencia de Néstor o Parrales, consigue finalmente hacerse respetar por su jefe e incluso se hacen amigos. En la última temporada podemos encontrar varios momentos en que Mauricio de forma cariñosa le llama "Machu". Y no es el único apodo. Otros personajes femeninos, como Soraya (Miren Ibarguren) y Macu (Pepa Rus), le llaman "Oswi". Néstor, de la misma serie, no tiene tanta suerte. Y en el caso de Parrales (La que se avecina), su jefe sólo le trata de forma adecuada desde el punto de vista personal y profesional cuando conviene a sus intereses económicos.

Otros ejemplos de Racismo vinculados a otros personajes se dan únicamente en la serie Aída. El propio Osvaldo se comporta de manera racista con sus compatriotas cuando les dice que no piensa viajar en Quitor Lines porque no se fía de que le lleve "un guachupino" (10×38). Soraya trata al principio al saudí Omar (Younes Bachir) como "morito" de manera despectiva (9×18) y la familia García al completo explotan a su invitado americano de procedencia asiática, Bobby Tang (Alberto Jo Lee), por considerar que los chinos pueden hacer de todo y, por tanto, se pueden aprovechar de él.

En definitiva, en estas series los momentos en que se aprecian elementos racistas son protagonizados por autóctonos, especialmente los personajes Mauricio Colmenero y Antonio Recio, propietarios de un pequeño negocio, y de ideología conservadora, machista y homófoba. Por otro lado, el caso del inmigrante Osvaldo, aunque esporádico, rompe con la dinámica general y con el estereotipo de víctima. 


\subsubsection{Violencia, Utilitarismo y Machismo}

En Aída el personaje con el que se puede vincular la Violencia en relación al racismo es Mauricio, cuyas prácticas con los folios o el estropajo ya han sido mencionadas. En La que se avecina, el caso de Antonio Recio destaca por la gravedad de los hechos. En primer lugar, Recio en un momento dado pretende matar a Parrales de un disparo por celos infundados: "Te has trincao a mi mujer y tienes que morir, son costumbres españolas" (4×12); y en segundo, al pensar por error que su abuelo era marroquí, se disfraza al estilo de los talibanes y planea "hacer explotar una bombona tirando balines con una escopeta de feria" $(5 \times 10)$.

En este caso, y a diferencia de lo que se ha afirmado en investigaciones anteriores, la Violencia está asociada a autóctonos además de a inmigrantes. Estos últimos están representados en mafias rumanas y narcotraficantes colombianos.

Con respecto al Utilitarismo, bien por motivos económicos o sexuales, son los autóctonos los protagonistas. EnLa que se avecina se puede mencionar, por referencia a la actualidad, el capítulo en el que Amador (Pablo Chiapella) cobra 3.000 euros a Parrales a cambio de casarse con él y de esa manera salir este último de la irregularidad.

En Aída, en relación al dinero, encontramos a Bobby Tang, el americano de origen asiático acogido en casa de la familia García porque el Estado otorgaba una paga por ello, y que finalmente se marcha de Madrid asustado y herido sin ninguna intención de volver, de ahí que el capítulo se llame "El último Tang en Madrid" (4×05). Además del factor económico, existen razones de tipo sexual. La joven Lorena es un ejemplo reiterativo de Utilitarismo con connotaciones sexuales. En afán de tener relaciones con hombres procedentes de todas partes del mundo, llega a insinuarse al saudí Omar cuando acaba de conocerle, comentando a su hermana Soraya: "De esos países todavía no he catao a ninguno [...] me da a mí que mañana por la noche me bajo al moro" $(9 \times 18)$.

Por último, el Machismo se genera por un interés utilitarista sexual y lo protagonizan autóctonos. Hay que destacar que las mujeres objeto de ese machismo son europeas, la española Mari Carmen novia de Parrales (La que se avecina) y Mary (Nicola Jones), una joven profesora inglesa (Aída).

Por lo que se puede comprobar, estas tres categorías tienen como máximos representantes a personajes españoles, y las víctimas son los inmigrantes; aunque esta afirmación está condicionada por el corpus analizado. En cualquier caso, habría que preguntarse si este hecho contribuye a crear una idea de superioridad de los españoles frente a los inmigrantes.

\subsubsection{Engaño, Chantaje y Subordinación}

Al igual que las categorías anteriores, el Engaño, el Chantaje y la Subordinación son Actos negativos. Los dos primeros están directamente relacionados con el Racismo, la Violencia y el Utilitarismo, ya sea porque son causa o consecuencia de los mismos. La Subordinación es el único rol pasivo que aparece como resultado de acciones de engaño, de violencia u otras; $y$ hemos querido analizarlo junto al Chantaje porque son las únicas categorías que pertenecen en exclusiva a los personajes inmigrantes.

En primer lugar, es notorio que, por un lado, el Engaño sea propio de los autóctonos, y por otro, el Chantaje de los inmigrantes, cuando ambos atributos tienen rasgos en común. Por un lado, el Engaño se lleva a cabo, además de por dinero, por amistad: Mauricio (Aída) engaña a Macu y Osvaldo robándoles los billetes de avión con destino a Ecuador para que se queden con él en España (10×38); y Maxi (Eduardo Gómez Manzano - La que se avecina) soborna al funcionario de turno para ayudar a Amador a casarse cuanto antes con Parrales: "Me ha costao 100 pavos untar al funcionario para que te cuele" $(4 \times 05)$.

Por otro lado, el Chantaje tiene como objetivo el dinero (destaca el papel estereotipado del cubano Oswaldo [Joel Angelino] que cambia sexo por dinero) pero también la dignidad, y en el caso concreto de los camareros de Aída, la defensa de sus derechos laborales. Aquí hay una distinción importante entre las series: mientras Osvaldo de Aída utiliza sus conocimientos y sus contactos para poner en un aprieto a su jefe y de esa manera conseguir lo que desea, Parrales no ejerce en ningún momento de líder y siempre le tienen que ayudar otros a conseguir mejoras en su trabajo, su salario o sus vacaciones. Su propia esposa el día de la boda intercede por él para poder tener el viaje de novios que Antonio Recio pretende boicotearles:

(Mari Carmen) Oiga, que Rosario tiene derecho a sus vacaciones. (Antonio) Qué queréis, hundirme el negocio. A que llamo a inmigración y os hacen una redada. Te quedas sin invitados. (Mari Carmen, muy cabreada) iiA que le denuncio yo al Ministerio de Trabajo por incumplimiento de contrato!! (Antonio) ¿Me estás amenazando? Eres una vergüenza para tu raza (Episodio "Parrales se casa").

En el papel opuesto a estas dos categorías se encuentra la Subordinación, protagonizada por inmigrantes. En el caso de Aída, son Osvaldo y Néstor los que tienen que agachar la cabeza siempre que su jefe les obligue a ello, aunque ya hemos visto que Osvaldo cobra cada vez más peso en las tramas y con ello también más seguridad y confianza en sí mismo. En esta serie también se encuentran los ya citados Bobby Tang, el americano-asiático; y el sueco Lars, al que Eugenia se lleva de compras para que le lleve todas las bolsas; y Jonathan le disfraza de torero para que salga a la plaza y haga reír a sus amigos: "Recuerda, cuando salga el segundo toro, tú saltas a la arena. Es tradición española" (8×14). Como en casos anteriores, utiliza la excusa de que es una costumbre cultural para lograr sus objetivos con aquéllos que la desconocen.

\subsubsection{Solidaridad}

Por último, se encuentra el único Acto con connotaciones positivas. Los personajes solidarios son principalmente autóctonos: el tendero deAída intenta siempre ayudar a los camareros latinoamericanos, el propio Mauricio Colmenero en un momento dado ayuda a unas prostitutas (algunas de las cuales son inmigrantes); en La que se avecina, Amador deja a Parrales dormir en su casa una noche y Berta intercede muchas veces por Parrales frente a su marido el pescadero. Pero no son sólo solidarios los autóctonos: Osvaldo, en las últimas temporadas en que ya tiene mayor protagonismo, ayuda a su compañero Néstor; y Luz Marina, que acaba en la cárcel por tráfico de drogas, salva más adelante a una de las vecinas de La que se avecina, Maite (Eva Isanta), de ser agredida sexualmente por unas presas.

\subsection{Significados asociados al Lenguaje}

El Lenguaje es una categoría esencial en el estudio de contenido, y en esta investigación en concreto, adquiere todavía mayor importancia porque está impregnado de Humor, característica básica de ambas comedias de situación, cuyo principal objetivo es precisamente burlarse y reírse de las convenciones sociales, de los estereotipos y, dentro del ámbito que estamos trabajando, del racismo. 
En primer lugar, destaca el personaje autóctono Luisma (Aída). Este hombre joven de unos 30 años, soltero, poco cualificado, con una formación educativa muy básica, y una inteligencia que se pone muchas veces en entredicho, es el alma del Humor absurdo. Cada vez que habla inserta un giro lingüístico cargado de humor. En relación a los inmigrantes, bromea sobre el americano Tang, Osvaldo y David el senegalés: "O sea que es ¡él! Bueno, yo mejor os dejo solos para que os aclaréis, ¿eh? Oye, que lo de aclarar no iba por lo de tu piel, ¿eh?" (7×10).

De vez en cuando también incluye alguna ironía, pero destaca especialmente por lo absurdo de sus gracias. Otro personaje relevante es Mauricio Colmenero, que tiene un humor muy diferente, caracterizado por la sátira: "Anda Machu, pásale el teléfono del jesuita que enseñó a hablar a tu tribu (ríe)" (9×14).

En La que se avecina predomina también el Racismo discursivo a través del lenguaje mordaz y la sátira. Algunas frases paradigmáticas son: "Los inmigrantes están preparados para sufrir vejaciones. ¡Yo soy español! Una raza de conquistadores" (4×11). "Éstos son alimentos de la jungla. Mi estómago no los digiere" (4×12). "Berta, para los inmigrantes nunca es fiesta. ¿Has visto alguna vez un chino de vacaciones?" (4×12). "Dile que se vuelva a su país que seguro que estará mejor" (episodio "Parrales se casa").

Por último, otra categoría relacionada con el Lenguaje y que es clave para entender los rasgos de una comedia de situación son los Tópicos, que aglutinan los estereotipos que se plasman sobre los inmigrantes. En Aída, del cubano Oswaldo se dice que, como caribeño, es impulsivo, pasional y huidizo. Por otro lado, las expectativas de la llegada del americano hacen soñar a Jonathan con una banda que le enseñe a burlar el detector de metales, a Lorena con un surfero rubio y cachitas, y a Luisma con un americano de las películas del oeste, con caballo y todo. Además de estos dos ejemplos tan evidentes, el sueco Stig parece recordar al tópico de los años 60 en España sobre la belleza de las suecas.

En el caso de La que se avecina, es característico el hecho de que Amador, al pensar en una colombiana, hable sobre "una mulata guapa y sexy"; o que Berta (Nathalie Seseña), para hacer ver a su marido que es "español de pura cepa" lo convierta en descendiente del Cid. Pero sin lugar a dudas, el tópico más paradigmático convertido en prejuicio es que la madre de Parrales, Luz Marina, de origen colombiano, sea una "mula", en otras palabras, transporte ilegalmente droga. Coque (Nacho Guerreros), el portero, ironiza al respecto, aunque dando la impresión de que lo dice en serio: "Claro, ha traído un producto típico de su tierra, como nosotros con el jamón" (episodio "La madre de Parrales, una mula").

\subsection{Participación}

A diferencia de otras series en que no hay Evolución en los personajes inmigrantes (son muy lineales), en este proyecto cabe señalar una transformación desde el punto de vista emocional y jurídico, respectivamente, en Osvaldo de Aída: de tener mujer y más de 10 hijos ecuatorianos, acaba ennoviándose con Macu, una española, yéndose a vivir con ella al final de la serie a Ecuador. Además, desde el punto de vista narrativo, de personaje secundario pasa a ser protagonista y una pieza clave en las tramas de la serie. En cuanto a Parrales de La que se avecina, es un personaje secundario en todas las tramas, pero desde el punto de vista legal, pasa de ser un "irregular" a casarse con una española, una mujer que además va a defender su dignidad y sus derechos laborales.

Por otro lado, sigue quedando patente que existe una Infrarrepresentación de los personajes inmigrantes que se detecta especialmente en un menor número de diálogos que los autóctonos. Sin embargo, la presencia de estos colectivos en las series pone de manifiesto, como se comentó en las primeras líneas de este trabajo, el incremento de esta población y la variedad cultural observada en la geografía española en la última década.

Aquí hay una diferencia sustancial entre las dos series: mientras que en La que se avecina la nacionalidad colombiana es la única a la que se otorga algo de protagonismo, en Aída son ocho nacionalidades diferentes. Por ejemplo, en esta última serie cabe destacar que los ingleses y marroquíes, que en España han ascendido de forma importante en cuanto a número de población, tienen presencia en varios capítulos. La aparición de Omar en los últimos años de Aída podría corresponderse con el aumento de población marroquí en la realidad. La población latinoamericana, por su parte, es la que más peso tiene a lo largo de toda la serie, y podemos reseñar el hecho de que en el último capítulo el personaje ecuatoriano se marcha a su país de origen, como están haciendo muchos de sus contemporáneos en la realidad en estos últimos años. Otra cuestión que es importante destacar es que, sin duda alguna, los rumanos son los grandes olvidados en estas series, siendo que en la actualidad en nuestro país constituyen el grupo inmigrante más numeroso $[3]$.

\subsection{Relaciones}

Las Relaciones están vinculadas a los sentimientos de unos personajes con otros, y constituyen una novedad en la representación de la inmigración en las series. El Amor y la Amistad se basan en relaciones interpersonales estrechas y que denotan una mayor vinculación de los personajes inmigrantes con el contexto y las tramas.

El Amor surge entre autóctonos e inmigrantes, lo que da lugar a relaciones interraciales e interculturales. Por un lado, Parrales con la ya mencionada María del Carmen. Y por otro, en Aída destaca la relación esporádica entre Lorena y Omar el saudí; la de Osvaldo con Macu, pareja que también hemos comentado; así como la propia Macu con David, el senegalés, con el que no vive un noviazgo, pero sí comparten la custodia del hijo que tienen en común.

En cuanto a la Amistad, es una categoría que predomina en Aída en el papel del camarero Osvaldo, que demuestra en los últimos momentos su buena relación con Mauricio, a pesar de todo, y con todos sus compañeros del bar, independientemente de su nacionalidad.

\subsection{Contextos}

Por último, esta macrocategoría hace referencia a la situación social, económica, cultural, etc.; que en este caso se atribuye al estatus laboral, social y educativo de los personajes. Así, se confirma que la representación clásica del inmigrante irregular y con baja cualificación predomina en las series estudiadas. Los ejemplos más característicos de Aída y La que se avecina son Osvaldo y Parrales, respectivamente, que no tienen papeles y tampoco estudios superiores que les permitan trabajar en otro ámbito distinto a la hostelería o al comercio.

Sin embargo, los personajes inmigrantes demuestran tener un conocimiento del mundo que les rodea superior a los autóctonos. Por ejemplo, el saudí Omar y Osvaldo, sin tener educación superior, manifiestan en muchos casos conocer mejor la cultura española que los autóctonos las costumbres musulmanas y la cultura ecuatoriana, o sencillamente conocimientos del mundo: "(Macu) Ay Oswi, ya le he dicho a mi madre que nos vamos a Ecuador. Y le parece perfecto. Lo único que me ha pedido es que vayamos los domingos a su casa (Osvaldo) Bueno (le acaricia con ternura) ya te lo explicaré en el vuelo trans-oceánico (Macu) ¿Ein? (10×38)". 


\section{CONCLUSIONES}

La inmigración en los últimos años ha sido un fenómeno que ha tenido importantes consecuencias demográficas, políticas, económicas y sociales; de ahí su trascendencia en los medios de comunicación, incluidas las series de ficción. En este contexto, es fundamental que seamos conscientes del poder que tienen los medios de transmitir y crear arquetipos. Por esta razón, es fundamental educar en el consumo de los mismos a la población, especialmente a los adolescentes, que viven una etapa de continuo desarrollo de su identidad. El presente trabajo pone de manifiesto la responsabilidad de los medios de comunicación en la formación de ciudadanos globales y críticos, en este caso ante el fenómeno migratorio y sus consecuencias.

En las series analizadas, Aída y La que se avecina, el retrato que se ofrece de los personajes inmigrantes sigue estando vinculado, como en investigaciones anteriores: a la violencia, el chantaje, la subordinación al poder del autóctono, una escasa participación como protagonistas de las tramas; una situación social, económica y cultural precaria; y además son irregulares o ilegales desde el punto de vista jurídico, lo que se traduce en el desempeño de trabajos poco cualificados con bajas remuneraciones. Además, se puede afirmar que en estas series sigue patente el estereotipo de escasa formación educativa del inmigrante. Sin embargo, se observa una distinción importante con respecto a otros estudios, y es que en numerosas ocasiones se pone de manifiesto la superioridad de conocimientos en cultura general por parte de los extranjeros con respecto a los autóctonos.

Por otro lado, se representan relaciones asimétricas entre inmigrantes y autóctonos, manifestando estos últimos talantes de superioridad y autoridad sobre los migrantes. En este sentido, cabe destacar la autocrítica que se hace en Aída y La que se avecina a los autóctonos. Por ejemplo, muestran a unos personajes españoles que manipulan, utilizan y engañan a los inmigrantes, e incluso manifiestan rasgos de machismo, características comúnmente asociadas a los extranjeros. También se pone de manifiesto el uso de la violencia laboral de los españoles sobre los inmigrantes. Pero sin duda la tónica dominante en las actitudes y el lenguaje de estos españoles es el racismo: la discriminación por motivos de raza, nacionalidad, religión o cultura está presente en las dos series objeto de estudio.

Otra cuestión de interés es la existencia de relaciones interculturales e interraciales, aunque sigue existiendo el tópico Norte-Sur, que se plasma en que los españoles tratan adecuadamente a los inmigrantes de países del Norte, e incluso de forma muy positiva, en una primera impresión; y a los del Sur los reciben con prejuicios. Latinoamericanos, árabes y africanos tienen más dificultades inicialmente para integrarse que los ingleses o los suecos. Sin embargo, cabe señalar una diferencia importante en Aída, y es el caso del camarero latinoamericano Osvaldo, que consigue con el paso de las temporadas revertir su estado de subordinado y establecer relaciones de igual a igual con los autóctonos. En otras palabras, el guión de esta serie, en contraste con La que se avecina, muestra una evolución psicológica y afectiva del inmigrante que le permite ganar confianza en sí mismo y le ayuda a enfrentarse a los que le discriminan.

En definitiva, el perfil del inmigrante en las dos series objeto de estudio podría definirse como el de una persona en situación irregular y sin cualificación ni estudios, que, sin embargo, con el paso del tiempo y una mayor relación con los españoles, puede llegar a conseguir mejorar su estatus social e integrarse en la sociedad; aunque debe seguir haciendo frente a la discriminación y el racismo, teniendo todavía un papel de subordinado o víctima con respecto al autóctono.

En este contexto, no se puede olvidar que Aída y La que se avecina son comedias de situación y, por tanto, el Humor impregna todas las situaciones en las que se ha definido este perfil del inmigrante. Es decir, toda actitud o lenguaje discriminatorio se realiza bajo el paraguas de la comedia; y la lectura que puede extraerse es que la intención de los guionistas puede ser denunciar el racismo y la xenofobia a través de la sátira y la parodia. Sin embargo, más allá del lenguaje discriminatorio cargado de ironía, la representación que se hace del inmigrante es en sí estereotipada y en algunos casos puede llegar a ser prejuiciosa dependiendo de la interpretación de dichos mensajes.

Por todo ello, es muy importante la educación de la población, y especialmente de los jóvenes, en nuevas competencias y destrezas de comprensión, de análisis y distanciamiento crítico frente a los mensajes mediáticos que reciben (Fernández y Ciércoles, 2006). Es por tanto una responsabilidad compartida de medios, familias y centros educativos (García y Aparici, 2009), así como de los investigadores en comunicación, seguir reflexionando sobre estas temáticas. No en vano, los medios de comunicación pueden llegar a afectar a las normas de comportamiento, socialización o construcción simbólica de la realidad de los jóvenes y, en definitiva, al futuro de nuestra sociedad.

\section{REFERENCIAS BIBLIOGRÁFICAS}

ANDRÉU, J. (2001): Las técnicas de Análisis de Contenido: Una revisión actualizada. Sevilla, Centro de Estudios Andaluces.

BARDIN, L. (1977): Análisis de contenido. Madrid, Akal Universitaria.

BISCARRAT, L.; MELÉNDEZ, N. (2014): "De la exclusión a la heteronomía. Inmigrantes en la ficción televisiva Aída”, Icono 14, n¹2, pp. 319346.

BAÑÓN, A. (2002): Discurso e inmigración. Propuestas para el análisis de un debate social. Murcia, Universidad de Murcia, Servicio de Publicaciones.

CASTLES, S.; DE HAAS, H.; MILLER, M. J. (2014): The age of migration. International population movements in the modern world. Nueva York, Palgrave McMillan.

COLEMAN, J.C. (1985): Psicología de la adolescencia. Madrid, Ediciones Morata.

DURKIN, K. (1985): Television, sex roles and children, a developmental social psychological account Milton Keynes, Open University Press.

ERIKSON, E. (1968): Identidad, juventud y crisis. Buenos Aires, Paidós.

FERNÁNDEZ, C.; CIÉRCOLES, I. (2006): Internet, una herramienta para la educación en valores. Il Jornadas sobre Educación Cívica y Democracia: Educación, medios de comunicación y civismo. Fundación Manuel Giménez Abad de Estudios Parlamentarios y del Estado Autónomico. [En línea]. 13 noviembre 2017. [Consulta: 13 noviembre 2017]

<http://www.fundacionmgimenezabad.es/images/Documentos/2007/20070125_26_epp_comunicacion_fernandez_romero_y_ciercoles_pereta_es_o.pdf> 
FIGUERAS, M. (2009): La adolescente activa. Las series de ficción españolas como ámbito de construcción intersubjetiva del yo. La mirada de Telemo, $n^{\circ}$ 2. [En línea]. 13 noviembre 2017. [Consulta: 13 noviembre 2017] <http://revistas.pucp.edu.pe/index.php/lamiradadetelemo/article/view/3542/3425>

FISKE, J. (1987): Television culture. London, Methuen.

GALÁN, E. (2006): La representación de los inmigrantes en la ficción televisiva en España. Propuesta para un análisis de contenido. Revista Latina de Comunicación Social nº 61. [En línea]. 13 noviembre 2017. [Consulta: 13 noviembre 2017] <http://www.ull.es/publicaciones/latina/200608galan.pdf>

GARCÍA MATILLA, A.; APARICI, R. (2009): La imagen. Análisis y representación de la realidad. Barcelona, Gedisa.

GARCÍA DE CASTRO, M. (2007): “Los movimientos de renovación en las series televisivas españolas”, Comunicar n 30, pp. 147-153.

GONZÁLEZ DE GARAY, B.; ALFEO, J.C. (2012): "Complejos de inferioridad y superioridad: Estudio comparado de la representación del personaje inmigrante en Falwty Towers y Aída, entre la xenofobia y la parodia", Revista Comunicación nº 10 (1), pp. 929-942.

IGARTUA, J. J.; BARRIOS, I.; ORTEGA, F. (2012): "Analysis of the image of immigration in prime time television fiction”,Comunicación y Sociedad n²5 (2), pp. 5-28.

INNERARITY, C.; ACHA, B. (2010): “Los discursos sobre ciudadanía e inmigración en Europa: universalismo, extremismo y educación”,Política y Sociedad, $n^{\circ} 47$ (2), pp. 63-84.

LACALLE, C. (2008): El discurso televisivo sobre la inmigración. Ficción y construcción de identidad. Barcelona, Ediciones Omega.

LÉVI-STRAUSS, C. (1965): Antropología estructural. Barcelona, Paidós.

LIROLA, M. (2013): Estudio de la construcción de las mujeres y menores inmigrantes en una muestra de noticias de radio. Tonos digital: Revista electrónica de estudios filológicos, $n^{\circ}$ 25. [En línea]. 13 noviembre 2017. [Consulta: 13 noviembre 2017] <

http://www.um.es/tonosdigital/znum25/secciones/estudios-22-mujeres_e_inmigrantes_en_la_radio.htm>

LOFLAND, J. (1971): Analyzing social settings: A guide to qualitative observation and analysis. Belmont, CA, Wadsworth.

MCMILLAN, J. H.; SCHUMACHER, S. (2005): Investigación educativa. Una introducción conceptual. Madrid, Pearson educación.

MCQUAIL, D. (2000): Introducción a la teoría de la comunicación de masas. Barcelona, Paidós.

MEDINA, P.; CONCEPCIÓN, L.; RODRIGO, M. (2008): "Niveles semánticos de las representaciones sociales de la inmigración subsahariana. Los sucesos de Ceuta y Melilla según ABC", Estudios sobre el Mensaje Periodístico, n 14, pp. 129-148.

MEDRANO, C.; AIERBE, A.; PALACIOS, S. (2008): "La dieta televisiva y los valores: un estudio realizado con adolescentes en la Comunidad Autónoma del País Vasco", Revista Española de Pedagogía, nº 239, pp. 65-84.

MONTERO, Y. (2006): Televisión, valores y adolescentes. Barcelona, Gedisa.

PERINAT, A. (1997): El desenvolupament en l'adolescéncia. Barcelona, Editorial UOC.

RAMOS, C. (1995): "Los medios de comunicación, agentes constructores de lo real”, Comunicar, nº 5, pp. 108-112.

RODRIGO, M.; MEDINA, P. (2009): “Los medios de comunicación en contextos interculturales”, Sociedad y Discurso, n 16, pp. 21-39.

RUIZ COLLANTES, X.; FERRÉS, J.; OBRADORS, M.; PUJADAS, E.; PÉREZ, O. (2006): "La imagen pública de la inmigración en las series de televisión españolas", Quaderns del CAC, n² 23-24, pp. 107-132.

RYAN, G. W.; BERNARD, H. R.: Data management and analysis methods. En DENZIN, N. K.; LINCOLN, Y. S. (2003):Collecting and interpreting qualitative materials. Thousand Oaks CA, Sage, pp. 259-309

VAN DIJK, T.A. (1997): Racismo y análisis crítico de los medios. Barcelona, Paidós.

VINUESA, M. L.: La encuesta. Observación extensiva de la realidad social. en BERGANZA, M.R.; RUIZ, J. A. (2005.): Investigar en comunicación. Guía práctica de métodos y técnicas de investigación social en Comunicación. Madrid, McGraw Hill, pp.177-205.

[1] El periodo señalado de 2005 a 2013 ha sido seleccionado por corresponder con los años de emisión de las series; y las nacionalidades que se mencionan por constituir las principales comunidades de inmigrantes en España a lo largo de estas fechas.

[2] Los porcentajes de alumnado inmigrante y autóctono de Zaragoza los proporcionó el Servicio Provincial de Educación. Se escogieron, partiendo de un muestro no probabilista (Vinuesa, 2005), dos centros que tenían datos similares de nacionales y extranjeros, un tercero con un $71,6 \%$ de inmigración, y otro con un $2 \%$. La elección de la muestra se justifica en el marco de un proyecto doctoral.

[3] La población rumana en España a 1 de enero de 2014 era de 728.253 personas, un poco por encima de la marroquí, la segunda comunidad inmigrante más numerosa con 717.992 habitantes (INE, 2014).

\section{BREVE SEMBLANZA DE LOS AUTORES:}

Ana Isabel Abad Villamor. Doctora en Comunicación por la Universidad San Jorge, ha realizado labores de docencia e investigación en dicha universidad. Actualmente, sigue colaborando con el grupo de investigación Migraciones, Interculturalidad y Desarrollo Humano, con quienes ha publicado artículos en revistas de impacto, así como ha participado en congresos nacionales e internacionales. Su línea de trabajo es el estudio 
de la imagen de la inmigración en los medios de comunicación y el análisis de audiencias y estudios de recepción con jóvenes y adolescentes, en los que se aborda el concepto de identidad y las relaciones interculturales.

Cayetano Fernández Romero. Doctor en Historia por la Universidad de Navarra, comenzó su actividad profesional en el Departamento de Historia y el Instituto de Ciencias para la Familia de dicha universidad. En la actualidad, es profesor en la Facultad de Comunicación y Ciencias Sociales, y en el Instituto Humanismo y Sociedad de la Universidad San Jorge (Zaragoza). Ha participado en congresos nacionales e internacionales, proyectos europeos y ha publicado obras relacionadas con la familia, el fenómeno migratorio actual y la innovación docente universitaria dentro del EEES.

- Recibido: 22/11/2017

- Aceptado: 11/03/2018

Ámbitos. Revista Internacional de Comunicación, n.40, edición de primavera, 2018 